APS

physics

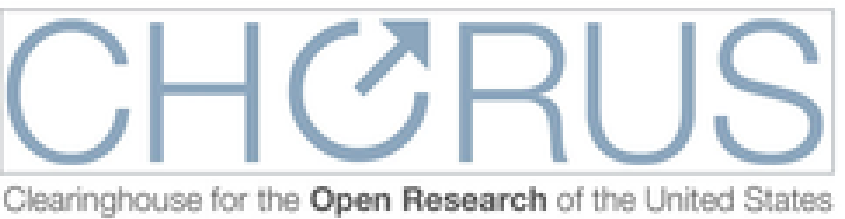

Clearinghouse for the Open Research of the United States

This is the accepted manuscript made available via CHORUS, the article has been published as:

\title{
Two-dimensional Potts antiferromagnets with a phase transition at arbitrarily large $q$
}

Yuan Huang, Kun Chen, Youjin Deng, Jesper Lykke Jacobsen, Roman Kotecký, Jesús Salas, Alan D. Sokal, and Jan M. Swart

Phys. Rev. E 87, 012136 - Published 24 January 2013

DOI: 10.1103/PhysRevE.87.012136 


\title{
Two-dimensional Potts antiferromagnets with a phase transition at arbitrarily large $q$
}

\author{
Yuan Huang, ${ }^{1, *}$ Kun Chen, ${ }^{1, \dagger}$ Youjin Deng, ${ }^{1, \ddagger}$ Jesper Lykke Jacobsen, ${ }^{2,3, \S}$

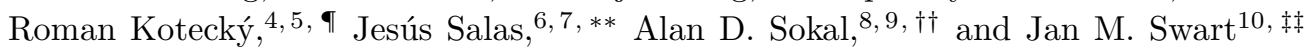 \\ ${ }^{1}$ Hefei National Laboratory for Physical Sciences at Microscale and Department of Modern Physics, \\ University of Science and Technology of China, Hefei, Anhui 230026, China \\ ${ }^{2}$ Laboratoire de Physique Théorique, École Normale Supérieure, 24 rue Lhomond, 75231 Paris, France \\ ${ }^{3}$ Université Pierre et Marie Curie, 4 place Jussieu, 75252 Paris, France \\ ${ }^{4}$ Center for Theoretical Study, Charles University, Prague, Czech Republic \\ ${ }^{5}$ Mathematics Institute, University of Warwick, Coventry CV4 7AL, UK \\ ${ }^{6}$ Escuela Politécnica Superior, Universidad Carlos III de Madrid, 28911 Leganés, Spain \\ ${ }^{7}$ Grupo de Teorías de Campos y Fúsica Estadística, Gregorio Millán Institute, \\ Universidad Carlos III de Madrid, Unidad asociada al IEM-CSIC, Madrid, Spain \\ ${ }^{8}$ Department of Physics, New York University, 4 Washington Place, New York, NY 10003, USA \\ ${ }^{9}$ Department of Mathematics, University College London, London WC1E 6BT, UK \\ ${ }^{10}$ Institute of Information Theory and Automation (ÚTIA), 18208 Prague 8, Czech Republic
}

\begin{abstract}
We exhibit infinite families of two-dimensional lattices (some of which are triangulations or quadrangulations of the plane) on which the $q$-state Potts antiferromagnet has a finite-temperature phase transition at arbitrarily large values of $q$. This unexpected result is proven rigorously by using a Peierls argument to measure the entropic advantage of sublattice long-range order. Additional numerical data are obtained using transfer matrices, Monte Carlo simulation, and a high-precision graph-theoretic method.
\end{abstract}

PACS numbers: 05.50.+q, 11.10.Kk, 64.60.Cn, 64.60.De

The $q$-state Potts model $[1,2]$ plays an important role in the theory of critical phenomena, especially in two dimensions (2D) [3-5], and has applications to various condensed-matter systems [2]. Ferromagnetic Potts models are by now fairly well understood, thanks to universality; but the behavior of antiferromagnetic Potts models depends strongly on the microscopic lattice structure, so that many basic questions about the phase diagram and critical exponents must be investigated case-by-case. In this article we prove the unexpected existence of phase transitions for some 2D $q$-state Potts antiferromagnets at arbitrarily large values of $q$.

For Potts antiferromagnets one expects that for each lattice $\mathcal{L}$ there is a value $q_{c}(\mathcal{L})$ [possibly noninteger] such that for $q>q_{c}(\mathcal{L})$ the model has exponential decay of correlations at all temperatures including zero, while for $q=q_{c}(\mathcal{L})$ there is a zero-temperature critical point. The first task, for any lattice, is thus to determine $q_{c}$.

Some 2D antiferromagnetic models at zero temperature can be mapped exactly onto a "height" model [6, 7]. Since the height model must either be in a "smooth" (ordered) or "rough" (massless) phase, the corresponding zero-temperature spin model must either be ordered or critical, never disordered. Until now it has seemed that the most common case is criticality [8].

In particular, when the $q$-state zero-temperature Potts antiferromagnet $(\mathrm{AF})$ on a 2D lattice $\mathcal{L}$ admits a height representation, one ordinarily expects that $q=q_{c}(\mathcal{L})$. This prediction is confirmed in most heretofore-studied cases: 3 -state square-lattice [6, 9, 12, 13], 3-state kagome $[14,15]$, 4-state triangular [16], and 4-state on the line graph of the square lattice $[15,17]$. Until recently the only known exception was the triangular Ising AF [18].

Kotecký, Salas and Sokal (KSS) [10] observed that the height mapping employed for the 3 -state Potts AF on the square lattice [6] carries over unchanged to any plane quadrangulation; and Moore and Newman [16] observed that the height mapping employed for the 4-state Potts $\mathrm{AF}$ on the triangular lattice carries over unchanged to any Eulerian plane triangulation (a graph is called Eulerian if all vertices have even degree). One therefore expects naively that $q_{c}=3$ for every (periodic) plane quadrangulation, and that $q_{c}=4$ for every (periodic) Eulerian plane triangulation.

Surprisingly, these predictions are false! KSS [10] proved rigorously that the 3 -state $\mathrm{AF}$ on the diced lattice (which is a quadrangulation) has a phase transition at finite temperature (see also [20]); numerical estimates from transfer matrices yield $q_{c}($ diced $) \approx 3.45[21]$. Likewise, we recently [11] provided analytic arguments (falling short, however, of a rigorous proof) that on any Eulerian plane triangulation in which one sublattice consists entirely of vertices of degree 4 , the 4 -state AF has a phase transition at finite temperature, so that $q_{c}>4$. We also presented transfer-matrix and Monte Carlo data confirming these predictions for the union-jack and bisected hexagonal lattices, leading to the estimates $q_{c}(\mathrm{UJ}) \approx 4.33$ and $q_{c}(\mathrm{BH}) \approx 5.40$.

These results suggest the obvious question: How large can $q_{c}$ be on a plane quadrangulation (resp. Eulerian plane triangulation)? The answers are clearly larger than 3 or 4 , respectively - but how much larger?

In this article we shall give a rigorous proof of the unexpected answer: we exhibit infinite classes of plane quad- 
rangulations and Eulerian plane triangulations on which $q_{c}$ can take arbitrarily large values. We shall also complement this rigorous proof with detailed quantitative data from transfer matrices, Monte Carlo simulations, and a powerful graph-theoretic approach developed recently by Jacobsen and Scullard [22].

The models studied here provide new examples of entropically-driven long-range order [10, 11, 20, 23]: the ferromagnetic ordering of spins on one sublattice is favored because it increases the freedom of choice of spins on the other sublattice(s). But though this idea is intuitively appealing, it is usually difficult to determine quantitatively, in any specific case, whether the entropic penalty for interfaces between domains of differentlyordered spins on the first sublattice is large enough to produce long-range order. Moreover, one expects that this penalty decreases with increasing $q$. In the examples given here, by contrast, we are able to prove that the penalty can be made arbitrarily strong and hence operative at arbitrarily large $q$.

The lattices $G_{n}$ and $H_{n}$. Let $G_{n}$ be obtained from from the square (SQ) lattice by replacing each edge with $n$ two-edge paths in parallel; and let $H_{n}$ be obtained from $G_{n}$ by connecting each group of $n$ "new" vertices with an $(n-1)$-edge path (see Fig. 1). Resumming over the spins on the "new" vertices [24], it is easy to show that the $q$-state Potts model on $G_{n}$ or $H_{n}$ with nearest-neighbor coupling $v=e^{J}-1$ is equivalent to a SQ-lattice Potts model with a suitable coupling $v_{\text {eff }}(q, v)$ [25]; moreover, for $q>2$ (resp. $q>3)$ an AF model $(-1 \leq v \leq 0)$ on $G_{n}$ $\left(\right.$ resp. $\left.H_{n}\right)$ maps onto a ferromagnetic model $\left(v_{\text {eff }} \geq 0\right)$ on the SQ lattice. Concretely, for the zero-temperature $\operatorname{AF}(v=-1)$ we have

$$
\begin{aligned}
& v_{\mathrm{eff}}^{G_{n}}(q,-1)=\left(\frac{q-1}{q-2}\right)^{n}-1 \\
& v_{\mathrm{eff}}^{H_{n}}(q,-1)=\frac{q-1}{q-2}\left(\frac{q-2}{q-3}\right)^{n-1}-1
\end{aligned}
$$

Setting $v_{\text {eff }}$ equal to the SQ-lattice ferromagnetic critical point $v_{c}(\mathrm{SQ})=\sqrt{q}[3,26]$, we obtain $q_{c}$ for $G_{n}$ and $H_{n}$; they have the large- $n$ asymptotic behavior

$$
q_{c}\left(G_{n}\right) \approx q_{c}\left(H_{n}\right) \approx \frac{2 n}{W(2 n)}+O\left((n / \log n)^{1 / 2}\right)
$$

where $W(x) \approx \log x-\log \log x+o(1)$ is the Lambert $W$ function [27]. We have thus exhibited two infinite families of periodic planar lattices on which the Potts AF has arbitrarily large $q_{c}$ as $n \rightarrow \infty$ [28]. These lattices are not triangulations or quadrangulations, but they can be modified to be such and retain the phase transition, as we now show.

The modified lattices. Starting from $G_{n}$ or $H_{n}$, insert a new vertex into each octagonal face and connect it either to the four surrounding vertices of the original SQ lattice, to the four "new" vertices, or to all eight vertices;

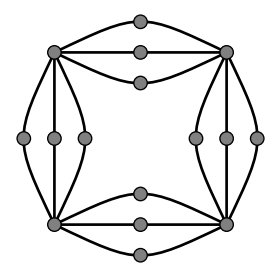

(a)

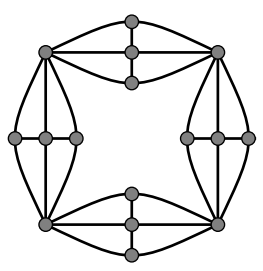

(b)
Figure 1. Unit cells of the lattices $G_{n}$ (a) and $H_{n}$ (b) for $n=3$.

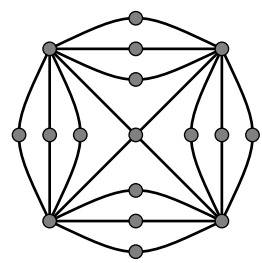

(a)

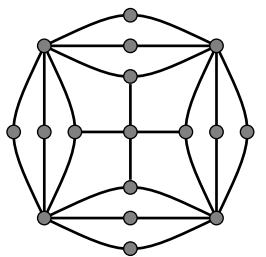

(b)

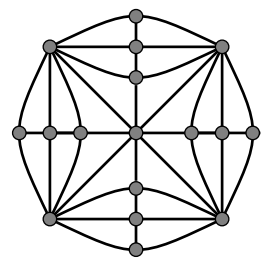

(c)
Figure 2. Unit cells of the lattices $G_{n}^{\prime}(\mathrm{a}), G_{n}^{\prime \prime}$ (b) and $H_{n}^{\prime \prime \prime}$ (c) for $n=3$.

call these modifications ', " and '", respectively. In particular, $G_{n}^{\prime}$ and $G_{n}^{\prime \prime}$ are quadrangulations, and $H_{n}^{\prime \prime \prime}$ is an Eulerian triangulation (Fig. 2).

If we integrate out the spins at the vertices placed into the octagonal faces, we obtain the model on $G_{n}$ or $H_{n}$ perturbed by a 4 -spin or 8 -spin interaction. When $q$ is large, this interaction is weak (of order $1 / q$ ) because its Boltzmann weight is bounded between a maximum value of $q$ and a minimum value of $q-4$ or $q-8$. We therefore expect that the new edges will have a negligible effect on the phase transition when $q$ is large, and that all the modified lattices will have $q_{c}(n)$ whose large- $n$ behavior is essentially identical to Eq. (3). Let us now sketch a rigorous proof [29] of this assertion.

Proof of phase transition. Recall first how one proves, using the Peierls argument, the existence of ferromagnetic long-range order (FLRO) at low temperature in the $q$-state Potts ferromagnet on the SQ lattice. The Peierls contours are defined as the connected components of the union of all bonds on the dual SQ lattice that separate unequal spins. A Peierls contour $\gamma$ of length $|\gamma|$ and cyclomatic number $c(\gamma)$ comes with a weight that is bounded above by $(q-1)^{c(\gamma)}(1+v)^{-|\gamma|}$ : here $(q-1)^{c(\gamma)}$ is a bound on the number of colorings of the SQ lattice consistent with the contour $\gamma$. Further, on the SQ lattice we have $c(\gamma) \leq|\gamma| / 2$, and the number of contours of length $n$ surrounding a fixed site can be bounded by $(n / 2) 16^{n}$. Standard Peierls reasoning then shows that for any pair of sites $x, y$ one has

$$
\operatorname{Prob}\left(\sigma_{x} \neq \sigma_{y}\right) \leq \sum_{n=4}^{\infty}(n / 2) 16^{n}(q-1)^{n / 2}(1+v)^{-n},
$$




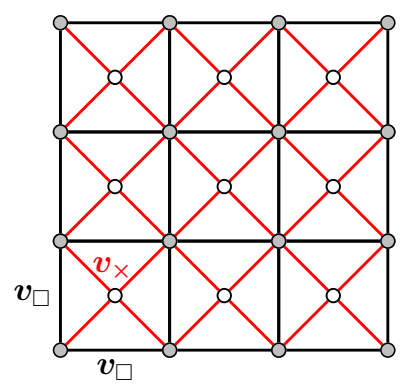

Figure 3. (Color online) The union-jack (UJ) lattice.

which is $\leq 5 / 16$ whenever $1+v \geq 32 \sqrt{q}$. This proves FLRO (the constant 32 is of course suboptimal). The foregoing argument is valid for fixed boundary conditions (e.g., $\sigma=1$ ) in the plane, but with suitable modifications it can also be carried out for periodic boundary conditions (i.e., on a torus).

Let us now consider the Potts antiferromagnet on one of the six modified lattices $G_{n}^{\prime}, \ldots, H_{n}^{\prime \prime \prime}$. Since our goal is to show FLRO on the SQ sublattice, we define Peierls contours exactly as we did for the SQ-lattice ferromagnet, ignoring the spin values at all other sites. Although we no longer have any simple explicit formula for the contour weights, it is nevertheless possible to prove an upper bound on the probability of occurrence of a contour $\gamma$ by using the technique of reflection positivity and chessboard estimates [30]. Without going into details of the needed adaptations of this standard technique for our case (see [29]), we mention only that the final bound on the probability of occurrence of a contour $\gamma$ is $(\kappa \sqrt{q-1})^{|\gamma|}$, where $\kappa$ is the probability that the spins on the SQ sublattice follow a fixed checkerboard pattern (say, 1 on the even sublattice and 2 on the odd sublattice) raised to the power $1 /$ volume. This latter probability is easy to bound explicitly, yielding $\kappa \leq$ $\left[1+v_{\text {eff }}(q, v)\right]^{-1}[q /(q-5)]^{1 / 2}$, where $v_{\text {eff }}(q, v)$ is the one for the corresponding unmodified lattice $G_{n}$ or $H_{n}$. This implies that, for all the lattices $G_{n}^{\prime}, \ldots, H_{n}^{\prime \prime \prime}$, there is FLRO on the SQ sublattice whenever $6 \leq q \leq q_{c}\left(G_{n}\right)-O(1)$ [cf. Eq. (3)] and $v$ is close to -1 (low temperature).

Let us also remark that the lattice $G_{2}^{\prime \prime}$ is covered by the general theory of [20], where it is proven that $q_{c}>3$; moreover, a minor modification proves the same result for $G_{n}^{\prime \prime}$ for all $n \geq 2$.

Data for lattices $G_{n}^{\prime}$ and $H_{n}^{\prime}$. The lattices $G_{n}^{\prime}$ and $H_{n}^{\prime}$ for all $n$ can be reduced to the union-jack (UJ) lattice (Fig. 3) with $v_{\times}=v$ and $v_{\square}=$ a suitable $v_{\text {eff }}(q, v)[\mathrm{cf}$. Eqns. (1)/(2) when $v=-1$ ]; of course the same reduction holds for $G_{n}$ and $H_{n}$ by setting $v_{\times}=0$. We obtained high-precision estimates of the phase boundary of the UJ model in the $\left(v_{\times}, v_{\square}\right)$-plane by using the JacobsenScullard (JS) method [22] with untwisted square bases of size up to $7 \times 7$ (294 edges) [31]. We checked these results for $q=4,8,16,32$ by Monte Carlo simulations

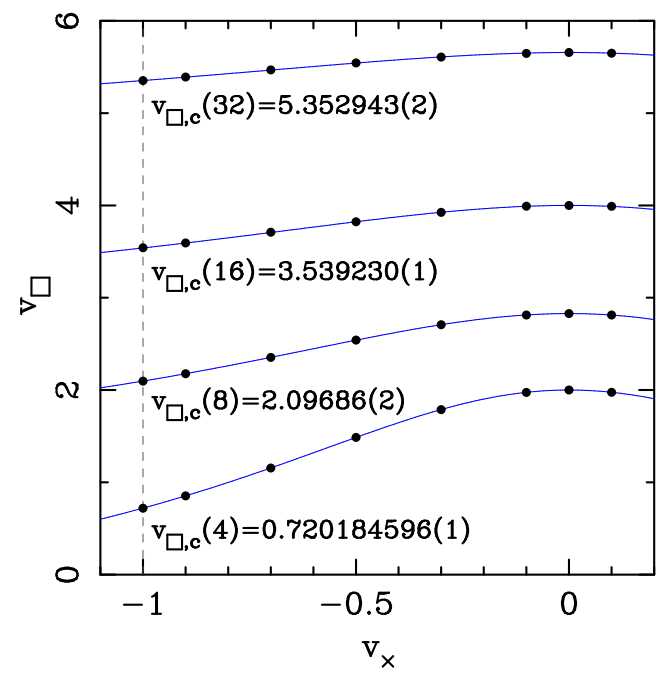

Figure 4. (Color online) Estimated phase boundaries for the $q=4,8,16,32$ Potts models on the union-jack (UJ) lattice, from the Jacobsen-Scullard method (blue curve and numerical values of $v_{\square, \mathrm{c}}$ at $v_{\times}=-1$ ) and Monte Carlo simulations (black points).

\begin{tabular}{|r||c|c|c|c||c|}
\hline \multicolumn{1}{|c||}{$n$} & $\begin{array}{c}q_{c}\left(G_{n}\right) \\
\text { (exact) }\end{array}$ & $\begin{array}{c}q_{c}\left(G_{n}^{\prime}\right) \\
(\mathrm{JS})\end{array}$ & $\begin{array}{c}q_{c}\left(H_{n}\right) \\
\text { (exact) }\end{array}$ & $\begin{array}{c}q_{c}\left(H_{n}^{\prime}\right) \\
(\mathrm{JS})\end{array}$ & $\begin{array}{c}2 n / W(2 n) \\
\text { (asymp. })\end{array}$ \\
\hline 1 & 2.618034 & $3.74583(8)$ & 2.618034 & $3.74583(8)$ & 2.345751 \\
2 & 3.448678 & $4.48805(4)$ & 4 & $4.80794(5)$ & 3.327322 \\
4 & 4.942152 & $5.87902(5)$ & 5.617069 & $6.39269(4)$ & 4.981903 \\
8 & 7.565625 & $8.40372(3)$ & 8.304127 & $9.04238(2)$ & 7.792741 \\
16 & 12.164794 & $12.91503(1)$ & 12.939420 & $13.63221(2)$ & 12.621338 \\
32 & 20.270897 & $20.945341(3)$ & 21.068717 & $21.711603(3)$ & 21.016077 \\
64 & 34.667189 & $35.276721(3)$ & 35.482095 & $36.074775(3)$ & 35.780223 \\
\hline
\end{tabular}

Table I. Estimates of $q_{c}(n)$ for the lattices $G_{n}, G_{n}^{\prime}, H_{n}, H_{n}^{\prime}$ from the Jacobsen-Scullard (JS) method or the exact solution, and their large- $n$ asymptote $2 n / W(2 n)$ from Eq. (3).

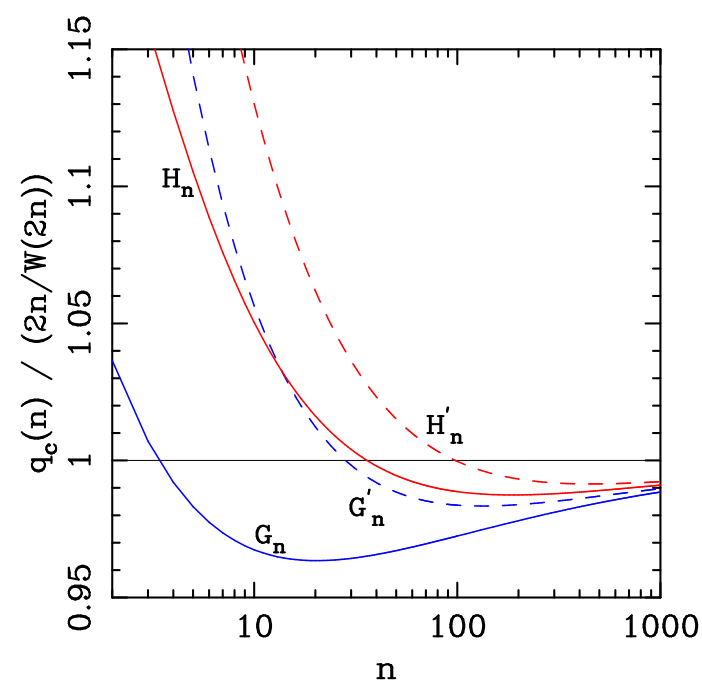

Figure 5. (Color online) Estimates of $q_{c}(n)$ for the lattices $G_{n}, G_{n}^{\prime}, H_{n}, H_{n}^{\prime}$ divided by their large- $n$ asymptote $2 n / W(2 n)$. 


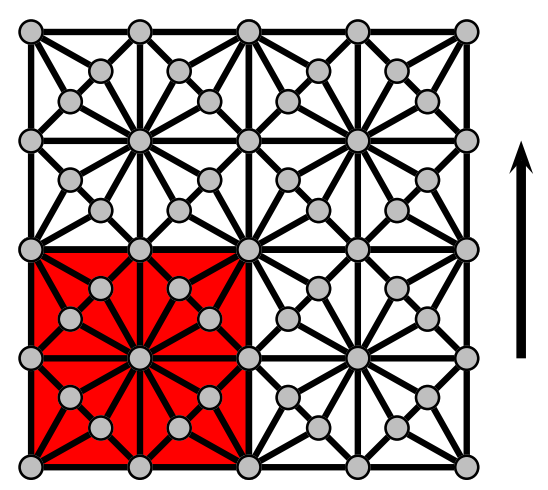

Figure 6. (Color online) The lattice $H_{2}^{\prime \prime \prime}$ (rotated $45^{\circ}$ from Fig. 2) with $L=2$; a unit cell is shown in red, and the transfer direction is indicated with an arrow.

using a cluster algorithm [32]. The estimated phase boundaries from both methods are shown in Fig. 4, along with the numerical estimates of $v_{\square, \mathrm{c}}$ at $v_{\times}=-1$ from the JS method. The estimates of $q_{c}(n)$ for the lattices $G_{n}, G_{n}^{\prime}, H_{n}, H_{n}^{\prime}$ from the JS method (or the exact solution) are shown in Table I, where they are compared with the predicted large- $n$ asymptote $q_{c}(n) \approx 2 n / W(2 n)$ from Eq. (3). The functions $q_{c}(n)$ divided by $2 n / W(2 n)$ are plotted in Fig. 5. Note that $q_{c}\left(G_{n}^{\prime}\right)>q_{c}\left(G_{n}\right)$ and $q_{c}\left(H_{n}^{\prime}\right)>q_{c}\left(H_{n}\right)$, in accordance with the intuitive idea that the AF edges associated to the modification ' enhance the ferromagnetic ordering on the SQ sublattice [33].

Data for lattices $G_{n}^{\prime \prime}$ and $H_{n}^{\prime \prime \prime}$. We studied the lattices $G_{n}^{\prime \prime}$ and $H_{n}^{\prime \prime \prime}$ for $n=1,2,4,8,16,32,64$ (note that $G_{1}^{\prime \prime}=$ SQ [6] and $H_{1}^{\prime \prime \prime}=\mathrm{UJ}$ [11]) at $v=-1$, using transfer matrices with cylindrical boundary conditions on widths $L=1,2,3,4$ unit cells (Fig. 6). The computational complexity is linear in $n$. We estimated the location of the phase transition (which we expect to be first-order whenever $\left.q_{c}>4\right)$ using the crossings of the energies $E_{L}(q)$ [34]: the results are shown in Table II.

For $H_{n}^{\prime \prime \prime}$ we checked these results by Monte Carlo: for three integer values of $q$ below the estimated $q_{c}$ we simulated the model at finite temperature and estimated the transition point $v_{c}(q)$; we then performed linear and quadratic extrapolations to locate the point $q_{c}$ where $v_{c}=-1$. The results are shown in Table II and Fig. 7 and agree well with the transfer-matrix estimates. For $q \gtrsim 8$ the specific heat diverges at the transition point like $L^{\approx 2}$, in agreement with the finite-size-scaling prediction for a first-order transition; for $4<q \lesssim 8$ the transition is presumably also first-order but with a large correlation length $\xi$, so that we are unable to observe the true $L \gg \xi$ asymptotic behavior.

Conclusion. When a 2D model admits a height representation, it must be either critical or ordered. Until now criticality seemed to be the most common case, even though examples of order were known. But here we have

\begin{tabular}{|r|||c||c|c||c|}
\hline \multicolumn{1}{|c||}{$n$} & $\begin{array}{c}q_{c}\left(G_{n}^{\prime \prime}\right) \\
(\mathrm{TM})\end{array}$ & $\begin{array}{c}q_{c}\left(H_{n}^{\prime \prime \prime}\right) \\
(\mathrm{TM})\end{array}$ & $\begin{array}{c}q_{c}\left(H_{n}^{\prime \prime \prime}\right) \\
(\mathrm{MC})\end{array}$ & $\begin{array}{c}2 n / W(2 n) \\
\text { (asymp. })\end{array}$ \\
\hline 1 & 3 & $4.31(3)$ & & 2.345751 \\
2 & $3.63(2)$ & $5.27(1)$ & $5.26(2)$ & 3.327322 \\
4 & $5.02(1)$ & $6.68(1)$ & $6.67(3)$ & 4.981903 \\
8 & $7.60(1)$ & $9.21(1)$ & $9.21(7)$ & 7.792741 \\
16 & $12.18(2)$ & $13.73(2)$ & $13.73(10)$ & 12.621338 \\
32 & $20.29(3)$ & $21.76(3)$ & $21.76(32)$ & 21.016077 \\
64 & $34.70(5)$ & $36.10(5)$ & $36.14(8)$ & 35.780223 \\
\hline
\end{tabular}

Table II. Estimates of $q_{c}$ for the lattices $G_{n}^{\prime \prime}$ and $H_{n}^{\prime \prime \prime}$ from transfer matrices (TM) and Monte Carlo (MC), and their large- $n$ asymptote from Eq. (3).

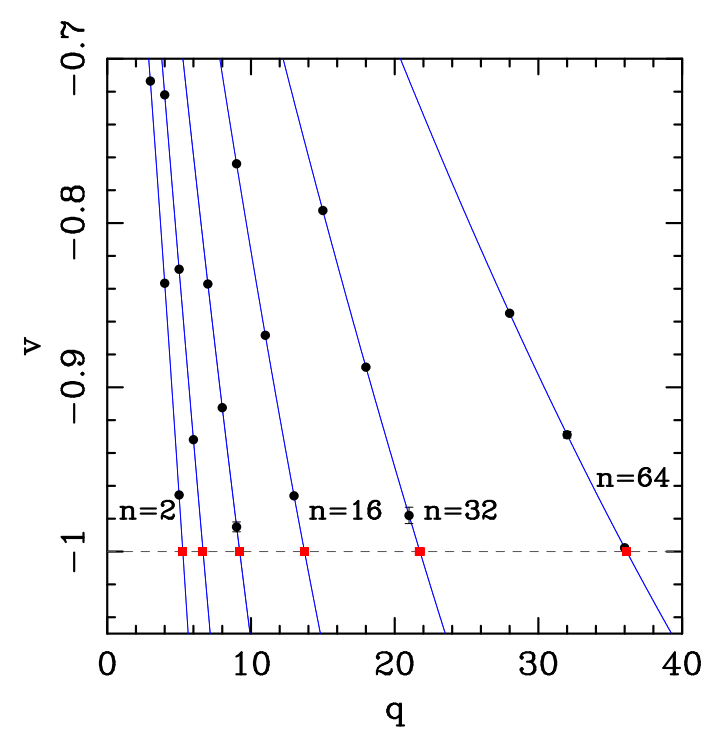

Figure 7. (Color online) Monte Carlo estimates of $v_{c}$ for the lattices $H_{n}^{\prime \prime \prime}$ (black points) and their quadratic fit (blue curves), together with the extrapolated values $q_{c}$ (red squares).

exhibited several infinite families of 2D lattices - some of which are quadrangulations or Eulerian triangulations - in which the Potts antiferromagnet admitting a height representation ( $q=3$ or 4 , respectively) is not only ordered but is in fact "arbitrarily strongly ordered" in the sense that $q_{c}$ is arbitrarily large. This unexpected result suggests that the prior belief may have things precisely backwards. Perhaps criticality is an exceptional case arising, for instance, in situations with special symmetries - and order is to be generically expected. A key open question raised by this work is to understand why criticality arises when it does.

This work was supported in part by NSFC grants 10975127 and 11275185, the Chinese Academy of Sciences, French grant ANR-10-BLAN-0414, the Institut Universitaire de France, Spanish MEC grants FPA2009-08785 and MTM2011-24097, Czech GAČR 
grant P201/12/2613, US NSF grant PHY-0424082, and a computer donation from the Dell Corporation.

* huangy22@mail.ustc.edu.cn

† chenkun@mail.ustc.edu.cn

‡ yjdeng@ustc.edu.cn

$\S$ jacobsen@lpt.ens.fr

ฯ R.Kotecky@warwick.ac.uk

** jsalas@math.uc3m.es

†† sokal@nyu.edu

$\ddagger \ddagger$ swart@utia.cas.cz

[1] R.B. Potts, Proc. Cambridge Philos. Soc. 48, 106 (1952).

[2] F.Y. Wu, Rev. Mod. Phys. 54, 235 (1982); 55, 315 (E) (1983); F.Y. Wu, J. Appl. Phys. 55, 2421 (1984).

[3] R.J. Baxter, Exactly Solved Models in Statistical Mechanics (Academic Press, London-New York, 1982).

[4] B. Nienhuis, J. Stat. Phys. 34, 731 (1984).

[5] P. Di Francesco, P. Mathieu and D. Sénéchal, Conformal Field Theory (Springer-Verlag, New York, 1997).

[6] J. Salas and A.D. Sokal, J. Stat. Phys 92, 729 (1998), cond-mat/9801079 and the references cited there.

[7] J.L. Jacobsen, in Polygons, Polyominoes and Polycubes, edited by A.J. Guttmann, Lecture Notes in Physics \#775 (Springer, Dordrecht, 2009), Chapter 14.

[8] Some exceptions are the constrained square-lattice 4state Potts antiferromagnet [9], the triangular-lattice antiferromagnetic spin- $s$ Ising model for large enough $s$ [C. Zeng and C.L. Henley, Phys. Rev. B 55, 14935 (1997), cond-mat/9609007], the diced-lattice 3-state Potts antiferromagnet [10], and the union-jack and bisectedhexagonal 4-state Potts antiferromagnets [11], all of which appear to lie in a non-critical ordered phase at zero temperature.

[9] J.K. Burton Jr. and C.L. Henley, J. Phys. A: Math. Gen. 30, 8385 (1997), cond-mat/9708171.

[10] R. Kotecký, J. Salas and A.D. Sokal, Phys. Rev. Lett. 101, 030601 (2008), arXiv:0802.2270.

[11] Y. Deng, Y. Huang, J.L. Jacobsen, J. Salas and A.D. Sokal, Phys. Rev. Lett. 107, 150601 (2011), arXiv:1108.1743.

[12] M.P.M. den Nijs, M.P. Nightingale and M. Schick, Phys. Rev. B 26, 2490 (1982).

[13] J. Kolafa, J. Phys. A: Math. Gen. 17, L777 (1984).

[14] D.A. Huse and A.D. Rutenberg, Phys. Rev. B 45, 7536 (1992).

[15] J. Kondev and C.L. Henley, Nucl. Phys. B 464, 540 (1996), cond-mat/9511102.

[16] C. Moore and M.E.J. Newman, J. Stat. Phys. 99, 629 (2000), cond-mat/9902295.

[17] J. Kondev and C.L. Henley, Phys. Rev. B 52, 6628 (1995); J.L. Jacobsen and J. Kondev, Nucl. Phys. B 532, 635 (1998), cond-mat/9804048.

[18] On the triangular lattice, both $q=2$ and $q=4$ are critical at zero temperature and have height representations
[19] [16], but $q_{c}=4$.

[19] H.W.J. Blöte and H.J. Hilhorst, J. Phys. A 15, L631 (1982); B. Nienhuis, H.J. Hilhorst and H.W.J. Blöte, J. Phys. A 17, 3559 (1984).

[20] R. Kotecký, A.D. Sokal and J.M. Swart, arXiv:1205.4472.

[21] J.L. Jacobsen and J. Salas, unpublished (2008).

[22] J.L. Jacobsen and C.R. Scullard, J. Phys. A 45, 494003 (2012), arXiv:1204.0622; C.R. Scullard and J.L. Jacobsen, J. Phys. A 45, 494004 (2012), arXiv:1209.1451; J.L. Jacobsen and C.R. Scullard, J. Phys. A (in press), arXiv:1211.4335.

[23] Q.N. Chen, M.P. Qin, J. Chen, Z.C. Wei, H.H. Zhao, B. Normand and T. Xiang, Phys. Rev. Lett. 107, 165701 (2011), arXiv:1105.5030.

[24] This is a special case of the Potts reduction formulae for 2-rooted subgraphs: see A.D. Sokal, in Surveys in Combinatorics, 2005, ed. B.S. Webb (Cambridge University Press, 2005), math.CO/0503607, Section 4.6.

[25] This equivalence was already observed in R. Kotecký, Phys. Rev. B 31, 3088 (1985) for the $d$-dimensional version of the lattice $G_{1}=H_{1}$ and used to show the longrange order for $q=3$ and $d \geq 3$.

[26] V. Beffara and H. Duminil-Copin, Probab. Th. Rel. Fields 153, 511 (2012), arXiv:1006.5073.

[27] This function is defined by $W(x) e^{W(x)}=x$ : see R.M. Corless et al., Adv. Comput. Math. 5, 329 (1996).

[28] A more complicated example with this property was given in the last paragraph of [10].

[29] R. Kotecký, A.D. Sokal and J.M. Swart, in preparation.

[30] J. Fröhlich and E.H. Lieb, Commun. Math. Phys. 60, 233 (1978); J. Fröhlich, R. Israel, E.H. Lieb and B. Simon, Commun. Math. Phys. 62, 1 (1978); M. Biskup, in Methods of Contemporary Mathematical Statistical Physics, ed. R. Kotecký (Springer, Berlin, 2009), math$\mathrm{ph} / 0610025$.

[31] We performed these computations for symbolic $q, v_{\times}, v_{\square}$ on bases up to $3 \times 3$ (54 edges), numerically for selected triplets $\left(q, v_{\times}, v_{\square}\right)$ near the phase boundary on bases up to $6 \times 6$ (216 edges), and numerically for $q=4, v_{\times}=-1$, $v_{\square} \approx v_{\square, \mathrm{c}}$ on bases up to $7 \times 7$ (294 edges). These results were made possible by an improvement of the transfermatrix method of [22] (J.L. Jacobsen, unpublished).

[32] The algorithm chooses randomly two of the $q$ colors and then simulates the induced mixed ferromagneticantiferromagnetic ( $\mathrm{F}-\mathrm{AF}$ ) Ising model by the SwendsenWang algorithm [R.H. Swendsen and J.-S. Wang, Phys. Rev. Lett. 58, 86 (1987)]. This is a slight extension of the WSK algorithm [J.-S. Wang, R.H. Swendsen and R. Kotecký, Phys. Rev. Lett. 63, 109 (1989); Phys. Rev. B 42, 2465 (1990)] to permit mixed F-AF couplings.

[33] This monotonicity would be a rigorous theorem if the Griffiths' first inequality of S.J. Ferreira and A.D. Sokal, J. Stat. Phys. 96, 461 (1999), cond-mat/9811345, Appendix A could be extended to a Griffiths' second inequality.

[34] C. Borgs, R. Kotecký and S. Miracle-Solé, J. Stat. Phys. 62, 529 (1991). 